\title{
The genetics of fat distribution
}

\author{
Dorit Schleinitz • Yvonne Böttcher • Matthias Blüher • \\ Peter Kovacs
}

Received: 1 November 2013 /Accepted: 18 February 2014 / Published online: 16 March 2014

(C) Springer-Verlag Berlin Heidelberg 2014

\begin{abstract}
Fat stored in visceral depots makes obese individuals more prone to complications than subcutaneous fat. There is good evidence that body fat distribution (FD) is controlled by genetic factors. WHR, a surrogate measure of FD, shows significant heritability of up to $\sim 60 \%$, even after adjusting for BMI. Genetic variants have been linked to various forms of altered FD such as lipodystrophies; however, the polygenic background of visceral obesity has only been sparsely investigated in the past. Recent genome-wide association studies (GWAS) for measures of FD revealed numerous loci harbouring genes potentially regulating FD. In addition, genes with fat depot-specific expression patterns (in particular subcutaneous vs visceral adipose tissue) provide plausible candidate genes involved in the regulation of FD. Many of these genes are differentially expressed in various fat compartments and correlate with obesity-related traits, thus further supporting their role as potential mediators of metabolic alterations associated with a distinct FD. Finally, developmental genes may at a very early stage determine specific FD in later life. Indeed, genes such as $T B X 15$ not only manifest differential expression in various fat depots, but also correlate with obesity and related traits. Moreover, recent GWAS identified several polymorphisms in developmental genes (including TBX15, HOXC13, RSPO3 and CPEB4) strongly associated with FD. More accurate methods, including cardiometabolic
\end{abstract}

Dorit Schleinitz and Yvonne Böttcher contributed equally to this study.

Electronic supplementary material The online version of this article (doi:10.1007/s00125-014-3214-z) contains peer-reviewed but unedited supplementary material, which is available to authorised users.

D. Schleinitz $\cdot$ Y. Böttcher $\cdot$ M. Blüher $\cdot$ P. Kovacs $(\square)$ Integrated Research and Treatment Center (IFB) AdiposityDiseases, University of Leipzig, Liebigstr. 21, 04103 Leipzig, Germany

e-mail: peter.kovacs@medizin.uni-leipzig.de

\section{Blüher}

Department of Medicine, University of Leipzig, Leipzig, Germany imaging, for assessment of FD are needed to promote our understanding in this field, where the main focus is now to unravel the yet unknown biological function of these novel 'fat distribution genes'.

Keywords Adipose tissue $\cdot$ Fat distribution $\cdot$ Genetics . GWAS
Abbreviations
CT Computerised tomography
eQTL Expression quantitative trait locus
FD Fat distribution
GWAS Genome-wide association studies
SNP Single nucleotide polymorphism
WC Waist circumference

\section{Introduction}

Obesity increases the individual risk for type 2 diabetes, dyslipidaemia, fatty liver disease, hypertension and cardiovascular disease [1]. However, obesity itself does not necessarily lead to these comorbidities [2-4]. Adipose tissue as a highly active endocrine organ is distributed at several body sites. As shown in early work by Kissebah [5] and Björntorp [6] on anthropometric correlates of fat distribution (FD) and clinical outcomes such as metabolic and cardiovascular diseases, fat stored in visceral adipose depots makes obese individuals more prone to these complications than fat distributed subcutaneously. The importance of visceral adipose tissue in the pathophysiology of insulin resistance, dyslipidaemia and cardiovascular disease was further supported by Matsuzawa et al [7] and Despres et al [8] in their pioneering work using imaging measurements of body FD. In this context, reduction in subcutaneous fat mass by liposuction does not ameliorate 
circulating metabolic and inflammatory variables [9]. On the other hand, visceral fat mass reduction by omentectomy combined with gastric banding results in long-term beneficial effects on glucose metabolism and insulin sensitivity [10]. The relationship of ectopic visceral fat deposition with metabolic and cardiovascular diseases may be at least partially explained by intrinsic properties of visceral as opposed to subcutaneous adipose tissue with regard to decreased insulin sensitivity, lower angiogenic potential, increased lipolytic activity, different cellular composition and the expression of genes regulating adipocyte function [11]. In addition, the visceral fat depot drains into the portal vein, thus exposing the liver to undiluted metabolites, cytokines and adipokines released from visceral fat, which could further contribute to an increased cardiometabolic risk [12]. Taken together, dysfunction of adipose tissue and ectopic fat seems to play an important role in the individual risk of developing obesityassociated metabolic and cardiovascular complications. However, it is noteworthy that recent imaging studies, including the Framingham Heart Study, have highlighted not only the importance of visceral adipose tissue, but also other ectopic fat depots such as liver or renal fat $[13,14]$.

\section{Measurement of fat distribution}

In clinical practice, waist circumference (WC) and WHR are widely used variables used to determine regional FD. Imaging techniques such as computerised tomography (CT) or whole body MRI scan are considered the gold standard for evaluating adipose tissue $[7,8]$. To measure the visceral and subcutaneous abdominal areas, a CT or MRI scan is usually taken at the level of L4-L5 or the umbilicus, using an attenuation range between -30 and -190 Hounsfield units [11]. The ratio of visceral to subcutaneous adipose tissue has been shown to be strongly correlated with impaired glucose and lipid metabolism in obese individuals [15]. This ratio, with a cut-off at 0.4 , allows stratification of obese individuals into visceral $(>0.4)$ and subcutaneous $(<0.4)$ groups [15]. Abdominal sagittal diameter derived from CT or MRI images has also been used to determine abdominal FD [11]. Applying CT scans in 403 volunteers, Lemieux et al proposed cut-off values corresponding to an accumulation of visceral adipose tissue of $130 \mathrm{~cm}^{2}$, which is strongly related to metabolic disorders: WHR of 0.94 in men and 0.88 in women, a waist girth of $\sim 95 \mathrm{~cm}$ and sagittal diameters of $22.8 \mathrm{~cm}$ in men and $25.2 \mathrm{~cm}$ in women [16]. It is noteworthy that, despite continuous technological advances in the measurement of FD, Lemieux et al have suggested the use of simultaneous measurement of WC and fasting triacylglycerol (hypertriglyceridaemic waist) as a simple screening tool to identify men characterised by the atherogenic metabolic triad (hyperinsulinaemia, elevated apolipoprotein B [ApoB], small, dense LDL particles) and at high risk for coronary artery disease [17].

\section{Which factors determine fat distribution?}

Together with genetic factors, the main predictors of visceral fat and FD are age, sex, total body fat content and energy balance [11]. Visceral fat mass increases with age independently of total body fat mass, but this is more pronounced in men than in women [11]. Sex plays a specific role, and this has also been reflected by differentiating obesity into android ('apple') and gynoid ('pear') types [18]. In this context, it has to be mentioned that sex hormones may modulate adipose tissue accumulation in a depot-specific manner. For example, declining oestrogen and/or excess androgens in postmenopausal women may facilitate fat partitioning from the periphery to the intra-abdominal cavity [19]. Alterations in endocrine signalling may also contribute to changes in FD. For instance, Cushing's syndrome is characterised by redistribution of fat from peripheral to central parts of the body [11]. Moreover, visceral fat mass in men is negatively associated with testosterone and sex-hormone binding globulin (SHBG) levels [20].

Despite facilitating the intra-abdominal accumulation of fat, a positive energy balance does not appear to be a major determinant of visceral fat mass. Energy intake/supply correlated with subcutaneous fat in monozygotic twins under hyper-energetic nutrition, yet the variation in visceral fat did not exceed $10 \%$ [21]. In contrast, there is good evidence that body weight loss results in an over-proportional reduction of the visceral fat mass [22], which might, at least in part, be explained by higher lipolytic capacity of visceral compared with subcutaneous fat [11].

In addition, it is likely that environmental factors either directly, or in genetically susceptible individuals, contribute to individual differences in FD (Fig. 1). For example, data from rodent studies suggest that food contaminants, particularly those with endocrine signalling capacities, may also cause ectopic fat accumulation in the liver and visceral fat depots [23]. There is also good evidence in humans that sugarsweetened beverages promote the accumulation of visceral adiposity [24]. Moreover, as suggested by Björntorp, stress mediated by psychosocial and socioeconomic handicaps, depressive and anxiety traits, alcohol and smoking may lead to neuroendocrine perturbations followed by abdominal obesity with its associated comorbidities [25].

\section{Genetic background of fat distribution}

There is good evidence that not only obesity but also FD is controlled by genetic factors, and that this is independent of 
Fig. 1 Genetics of FD: strategies to identify genes involved in fat distribution and potential mechanisms contributing to variability in FD. GWAS provide a major tool for identification of novel genes associated with FD measures, such as WHR. Candidate gene strategies rely on the investigation of genes with fat depot-specific mRNA expression. In addition, genes known to be involved in the pathophysiology of other forms of altered fat distribution, such as lipodystrophies, are potential candidates (e.g. $L M N A$ ). Developmental genes represent a unique group of genes that is not only supported by their physiological role but also by GWAS. $C B 1 R$ is also known as CNR1

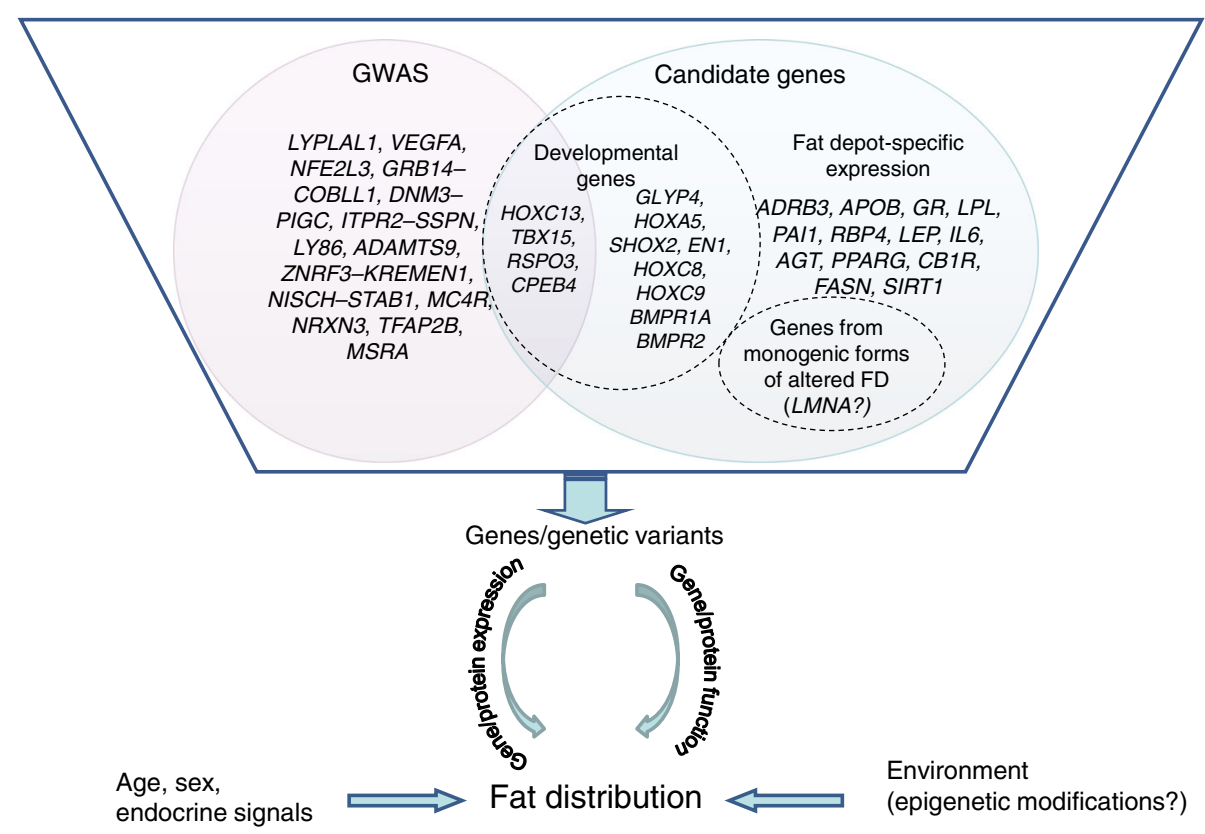

BMI and overall obesity [26, 27]. Studies estimating heritability of WHR have provided eminent proof for a genetic background to FD, showing that this trait is heritable with estimates ranging from $22 \%$ to $61 \%$ even after accounting for obesity [26-30]. In one of the pioneering works in this field, Bouchard et al showed in identical twins that within-pair similarity was particularly evident for changes in regional FD and amount of abdominal visceral fat, with significantly greater variance among pairs than within pairs, thus strongly suggesting the involvement of genetic factors [21]. In the Quebec Family Study, heritability estimates reached 56\% for abdominal visceral fat, while only $42 \%$ heritability was estimated for subcutaneous fat [31]. These data suggested a strong genetic influence on the familial aggregation in abdominal fat, independent of total body fat mass, and clearly indicated that genetic factors seem to have a greater effect on abdominal visceral fat than on abdominal subcutaneous adipose tissue. Supporting the earlier studies, heritability estimates of between $83 \%$ and $86 \%$ for total fat, and between $71 \%$ and $85 \%$ for regional fat (trunk, lower body, trunk/lower body) have been reported in young and elderly Danish twins [32]. Considering that variation in FD during adolescence is correlated with FD in adulthood, Peeters et al estimated the relative contribution of genes and environment to the stability of subcutaneous FD from early adolescence into young adulthood in the Leuven Longitudinal Twin Study [33] and showed that the distribution of trunk to extremity adipose tissue is under strong genetic control $(>75 \%)$ in boys and girls.

Since some individuals are genetically predisposed to store abdominal fat in the visceral rather than in the subcutaneous depot, the above-mentioned studies also implied that these individuals are at higher genetic risk of manifest metabolic complications associated with visceral obesity [31].

\section{Conditions of altered fat distribution}

Conditions such as steatopygia and lipodystrophies also support the role of genetics in FD. There are obvious differences in body FD as humans gain or lose weight. This is extremely profound in certain ethnic groups such as the Khoikhoi (previously known as Hottentots) in southern Africa, whose women show excessive accumulation of fat in the buttocks [34]. Lipodystrophies with abnormal regional fat deposition provide further convincing evidence for the role of genetics in FD [35]. For instance, for congenital generalised lipodystrophy, which is characterised by a partial or complete loss of any adipose tissue, mutations in four genes, AGPAT2, BSCL2, $C A V 1$ and $P T R F$, leading to disturbances in either lipid storage (AGPAT2) or lipid homoeostasis (CAV1, PTRF, BSCL2) have been postulated to be causative [36-39]. One of the most prominent genes causing familial partial lipodystrophy encodes lamin $\mathrm{A} / \mathrm{C}(L M N A)$ whose mutations result in familial partial lipodystrophy of the Dunnigan type, which is characterised by a loss of subcutaneous adipose tissue in the extremities and trunk, without loss of visceral, neck, or facial fat $[40,41]$. Patients with Dunnigan-type familial partial lipodystrophy suffer post puberty from regional and progressive adipocyte degeneration, which is often accompanied by profound insulin resistance and diabetes [42]. It has been demonstrated in mice that, rather than involving a loss of fat, the major mechanism contributing to the lack of fat 
accumulation is likely to be an altered renewal capacity of the adipose tissue, which could be attributed to the disturbed differentiation of pre-adipocytes into functional adipocytes [43]. Interestingly, common variants in LMNA have been shown to contribute to the polygenic background of type 2 diabetes and obesity [44-46], making $L M N A$ a plausible candidate for involvement in the pathophysiology of visceral obesity (Fig. 1).

Familial multiple lipomatosis is another condition of altered FD that is characterised by the presence of multiple lipomas on the body. Although an autosomal-dominant inheritance has been proposed [47], information on the genetic background of lipomatosis is sparse and research has so far focused on $H M G A 2$ and its fusion partners $L P P$ and $L H F P$ [48-50]. Of note, transgenic mice expressing truncated Hmga2 still retaining the three AT-hook domains are characterised by a giant phenotype and hyperplasia of white adipose tissue [51] whereas, on the other hand, HMGA2 knockout mice present a pygmy phenotype with hypoplasia of white adipose tissue [52]. Likewise, a lack of $H M G A 2$ impairs lineage commitment of stem cells toward preadipocytes, further supporting the role of $H M G A 2$ in adipogenesis [53].

\section{Candidate genes for regulating fat distribution}

The classical approach to examining the heterogeneity of adipose tissue is based on comparisons of protein and gene function and expression between the visceral and subcutaneous fat depots. Differential gene expression between visceral and subcutaneous adipose tissue points to genetic heterogeneity and, therefore, its investigation represents a promising path to reveal candidate genes involved in the regulation of FD. Indeed, there are numerous genes with differential expression between visceral and subcutaneous adipose tissue, such as $A D R B 3$ [54], $A P O B$ [55], GR (also known as NR3C1) [56], LPL [57], PAI1 [58], RBP4 [59], LEP [60], IL6 [61], AGT [60] or PPARG [62] (Fig. 1). It has been postulated that genetic variants in these genes may contribute to ectopic visceral storage [63]. Moreover, many of these genes, notably $A D R B 3$, $A P O B, L P L, R B P 4, L E P, I L 6, A P M 1$ and PPARG, are not only differentially expressed in various fat depots, but are also associated with traits related to obesity, such as insulin resistance or adipokine levels. Even though it is unclear whether differential expression is a cause or consequence of obesity and/or FD pattern, it might be postulated that changes in gene expression or function caused by genetic variants may link metabolic alterations to obesity. Consistent with this hypothesis, it was demonstrated that polymorphisms in genes such as FASN, BMPR1A, BMPR2 and RBP4 were associated not only with WHR and BMI-related traits but also with their transcript levels, indicating that the single nucleotide polymorphisms'
(SNPs') effects are mediated through regulation of gene expression [64-67]. These findings clearly indicate potential functionality of the identified polymorphisms in the regulation of FD. It has to be acknowledged, however, that in general, early candidate gene studies were mostly underpowered and the genotype-phenotype associations would not have withstood the currently accepted genome-wide statistical significance levels. Nevertheless, they undoubtedly deserve attention as they may still represent very promising targets contributing to a better understanding of the complex aetiology of obesity-related complications, and might even pave the way for novel treatment strategies in metabolic disorders.

For example, given its biological role in metabolism, PPARG (the gene encoding peroxisome proliferatoractivated receptor $\gamma$ ) is one of the most prominent candidates for involvement in modulating FD. Treatment of type 2 diabetes with thiazolidinediones, which activate PPARG selectively, increases fat partitioning to the subcutaneous adipose depot [68] and may also reduce visceral fat volume [69]. Therefore, it may not be surprising that the genetic variant predicting a Pro115Gln change in PPARG has also been investigated intensively in relation to FD and was not only found in extremely obese subjects but also shown to promote adipocyte differentiation [70]. In contrast, the Pro12Ala variant in PPARG is associated with lower BMI, better insulin sensitivity and reduced risk of type 2 diabetes [63]. Although a significant $P P A R G$ gene-sex interaction was observed in the modulation of BMI, fat mass and blood pressure for Pro12Ala, it was also associated with WC independently of BMI and sex [71]. Consistently, the polymorphism was associated with WHR and visceral and subcutaneous fat mass in Korean women, although the data suggested that the gene has a larger impact on subcutaneous than visceral adipose tissue [72].

As sirtuin 1 (SIRT1) is an important regulator of energy metabolism through its impact on glucose and lipid metabolism, genetic studies have been performed to test the effects of genetic variation in SIRT1 on adiposity. A Belgian casecontrol association study involving 1,068 obese patients and 313 lean controls suggested that genetic variants of SIRT1 increase the risk for obesity, and that the SIRT1 genotype correlates with visceral obesity variables (WC, WHR and visceral and total abdominal fat) in obese men [73].

\section{Genome-wide association studies}

By using the latest advances in high-throughput technologies as well as statistical approaches for well-powered genomewide association studies (GWAS), numerous novel and, from a biological point of view, unexpected genes/loci have been identified over the last couple of years. One of the earliest studies revealed an association between rs12970134 and WC 
in individuals of Indian-Asian or European ancestry [74]. The SNPs have been mapped near $M C 4 R$, which is known to be predominantly involved in monogenic obesity [74]. Since then, $M C 4 R$ has remained one of the major WC-associated loci, conferring a relatively large effect size of $0.88 \mathrm{~cm}$ per risk allele [74]. In addition, a few other loci related to WC, including the neurexin 3 gene (NRXN3), have been discovered [75]. Since the effect of the associated variant was markedly attenuated when adjusting for BMI, it is likely that NRXN3 is involved in regulating overall obesity rather than WC [75], which is in line with previous studies showing NRXN3, to be related to obesity and BMI [76].

The first meta-analysis of GWAS relating to WC and WHR was conducted by Lindgren et al and suggested a role for genetic factors in the regulation of both WC and WHR [77]. Genetic variants within TFAP $2 B$ and near MSRA were strongly associated with visceral fat accumulation (WC). Furthermore, the authors identified variants $\sim 250 \mathrm{~kb}$ downstream of LYPLAL1 with strong sex-specific variability, as associations with WHR were restricted to women only. In line with the Lindgren meta-analysis, a recent study conducted with 32 GWAS for WHR adjusted for BMI (up to 77,167 participants), and following up 16 loci in an additional 29 studies (up to 113,636 participants), uncovered 13 loci associated with WHR (RSPO3, VEGFA, TBX15-WARS2, NFE2L3, GRB14COBLL1, DNM3-PIGC, ITPR2-SSPN, LY86, HOXC13, ADAMTS9, ZNRF3-KREMEN1, NISCH-STAB1, CPEB4) and confirmed the known association signal at $L Y P L A L 1$, with effect sizes reaching 0.059 per risk allele in women (Fig. 2; electronic supplementary material [ESM] Table 1) [30, 77]. A recent GWAS including up to 263,407 individuals of European ancestry replicated associations with WHR for RSPO3, LY86, LYPLAL1 and COBLL1 [78]. Altogether, the GWAS findings indicate a strong genetic background for WHR regulation, independently of overall obesity.

In addition to GWAS for WHR, several studies used more precise measures of FD, such as visceral and subcutaneous fat area measured by CT $[79,80]$. These GWAS revealed additional variants implying the value of more accurate measurements in unravelling novel polymorphisms contributing to the genetic control of FD. In particular, Fox et al provided strong evidence for an association of a novel locus with visceral adipose tissue near THNSL2 in women [80]. Moreover, using the ratio of visceral to subcutaneous adipose tissue, significant associations were replicated for seven of the previously reported WHR loci (after adjusting for BMI) [30, 80]. Given the known limitations of WHR as a measure of FD, particularly based on the fact that, for a given WHR value, there may be large variation in the level of abdominal visceral adipose tissue, the data from Fox et al clearly demonstrate the need for including more accurate phenotypes of regional FD in future genetic analyses.

Although most of the previous GWAS were conducted in cohorts of European ancestry, recent studies have replicated the previously identified associations in various ethnic populations [81, 82]. While confirming six of the 14 loci described above for WHR (TBX15-WARS2, GRB14, ADAMTS9, LY86, RSPO3, ITPR2-SSPN), two novel regions have been shown to associate significantly with $\mathrm{WC}$ and WHR (LHX2 and $R R E B 1$, respectively; both adjusted for BMI) in individuals of African ancestry [81]. Furthermore, recent analyses of the 14 WHR loci confirmed the potential role in FD for LYPLAL1 and NISCH in a Japanese population [82].

It is of note that a recent GWAS identified a novel SNP near TRIP2 associated with pericardial fat [83]. The variant rs10198628 was exclusively associated with pericardial fat in a multi-ethnic survey, without any further evidence of association with visceral adipose tissue or BMI. The authors provided further evidence for an expression quantitative trait locus (eQTL) suggesting that the association of the lead variant close to TRIP2 might be mediated by its altered gene expression [83].

Sexual dimorphism in GWAS The fact that seven of the loci identified by Heid et al (RSPO3, VEGFA, GRB14, LYPLAL1, ITPR2-SSPN, ADAMTS9, HOXC13) (Fig. 2) exhibited significantly stronger effects on WHR in women than in men might explain why all the 14 loci explained $1.34 \%$ of the variance in WHR in women but only $0.46 \%$ of the variance in men [30]. The sexual dimorphism in FD gained further support from a very recent large-scale study comprising 133,723 individuals in the initial meta-analysis of GWAS and 137,052 individuals in subsequent replication stages [84]. Specifically, significant sex-related differences were replicated for four of the 14 previously reported WHR-associated loci (adjusted for BMI) near GRB14-COBLL1, LYPLAL1SLC30A10, VEGFA and ADAMTS9 (Fig. 2) [30, 84]. Moreover, three novel loci were identified for WC (near $M A P 3 K 1$ ) and WHR (adjusted for BMI) (near HSD17B4 and PPARG) (Fig. 2), whereas no evidence for sex-specific differences was found for other anthropometric measures. The observed sexual dimorphism may not be surprising when considering that sex differences manifest not only in WHR per se but also in the extent of genetic effects influencing variation in body composition. Although there is a shortage of studies systematically investigating sex differences in the genetic architecture of body composition, Zillikens et al showed that genetic variance was significantly higher in women for waist, hip and WHR in the Erasmus Ruphen Family study, thus suggesting that genes account for more phenotypic variance of FD in women than in men [85]. The above-mentioned GWAS strengthen the conclusions of the Erasmus Ruphen Family study and clearly imply the tremendous importance of sexspecific analyses in studies aimed at pinpointing the genetic architecture of complex traits such as FD. Nevertheless, it has to be kept in mind that genetic determinants of body composition may be modulated by sex-specific hormonal, 
Fig. 2 Sex-specific effect sizes of WHR-associated loci: effect sizes of all genome-wide significant WHR loci meta-analysed by (1) Heid et al [30] and by (2) Randall et al [84]. The data are ordered by effect sizes in women and reported for the combined stages of analyses. (3) Genome-wide significance in women; (4) genome-wide significance in men; black bars, women; white bars, men

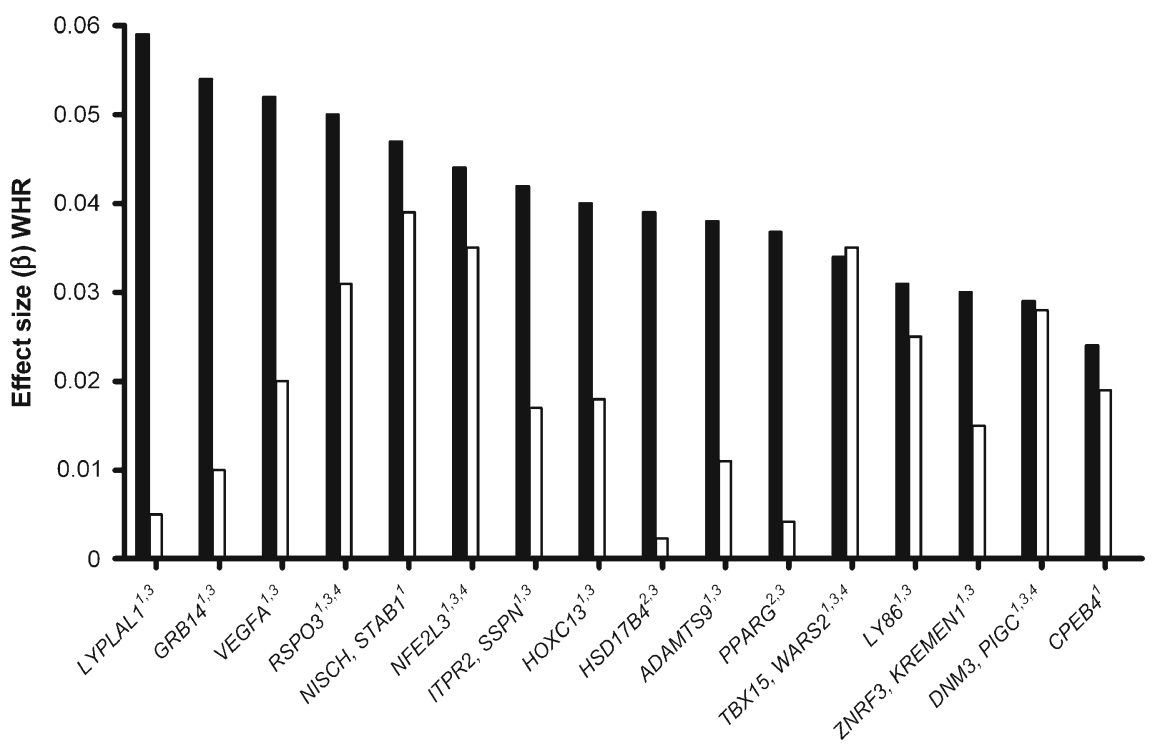

environmental and nutritional factors [85], which may at least partially explain the observed sex-related differences in genetic effects on FD.

Functional evidence for the role of genes identified by GWAS In contrast to the variants related to obesity and BMI, which are mostly expressed in the brain, the vast majority of the WHR-related genes identified by GWAS are predominantly expressed in peripheral tissues [76, 86, 87]. However, a functional role could be assigned to only a handful of candidate genes, predominantly involved in adipogenesis (TBX15), early embryonic development (HOXC13), angiogenesis (VEGFA, RSPO3 and STAB1), lipase activity (LYPLAL1) or lipid biosynthesis $(P I G C)$ (reviewed in [30]). With regard to the potential function of the genes identified, and apart from the sex-specific association signals, another important aspect of the GWAS carried out by Heid et al was the identification of eQTLs for six WHR-associated SNPs (rs984222 for TBX15 mRNA in omental adipose tissue; rs1055144 for AA553656 mRNA, rs10195252 for GRB14 mRNA and rs4823006 for ZNRF3 mRNA in subcutaneous adipose tissue; rs1011731 for $P I G C$ mRNA in lymphocytes; rs6784615 for STAB1 mRNA in blood) [30]. At these loci, the WHR-associated SNPs explained the majority of the association between the most significant eQTL and the gene transcript. Moreover, mRNA of the five potential FD-related genes (RSPO3, TBX15, ITPR2, WARS2 and STAB1) was differentially expressed between gluteal and abdominal subcutaneous adipose tissue. In follow-up studies, these data could be strengthened by demonstrating fat depot-specific differences in mRNA expression between subcutaneous and visceral adipose tissue for all six genes mapped within the reported eQTLs [88]. Whereas only $P I G C, Z N F R 3$ and STAB1 mRNA expression in subcutaneous adipose tissue correlated nominally with WHR, mRNA expression of all six genes in at least one of the fat depots correlated significantly with visceral and/or subcutaneous fat area. Finally, the rs 10195252 T-allele was nominally associated with increased GRB14 subcutaneous mRNA expression, suggesting that the association with WHR might be mediated by the SNP effects on mRNA expression levels. GRB14 appears to be an appealing gene as it binds to the insulin receptor and its expression is enhanced in patients with type 2 diabetes. Moreover, Grb14-deficient mice $\left(\mathrm{Grbl4}^{-/-}\right)$ manifest enhanced insulin signalling in liver and skeletal muscle, improved glucose homeostasis and increased body weight, mainly due to increased lean mass, on a normal diet $[89,90]$. When put on a high-fat diet, the $\mathrm{Grb} 14^{-1-}$ mice showed an increase in fat mass, detected by dual-energy X-ray absorptiometry (DEXA), which was not, however, accompanied by a parallel increase in epididymal fat mass [91]. Consequently, as postulated by Holt et al, it could either be the case that small differences in numerous adipose depots may lead to a significant overall difference in fat mass or, alternatively, that there may be depot-specific differences in fat accumulation with no change observed for the epididymal depot [91].

\section{Developmental genes in the regulation of fat distribution}

Fat depot-specific expression of developmental genes provides further support for the strong genetic background of FD [92]. It has been observed in both rodents and humans that visceral adipose tissue is characterised by higher mRNA levels of HoxA5, HoxA4, HoxC8, Gpc4 and Nr2f1, whereas subcutaneous fat has higher levels of HoxA10, HoxC9, Twist1, Tbx15, Shox2, En1 and Sfpr2. Even more importantly, such variability in gene expression is also found in pre-adipocytes 
derived from different fat depots in rodents and humans, and appears to be intrinsic, since it persists during in vitro culture and differentiation $[92,93]$. This may suggest that different mesodermal regions might give rise to precursors in different adipose depots, and might so contribute to biological differences between visceral and subcutaneous adipose tissue. In support of this, Gesta et al have shown that mRNA expression profiles of Tbx15, Gpc4 and HoxA5 not only differ between various adipose depots but also strongly correlate with BMI, WHR or visceral fat mass and subsequent metabolic alterations in mice as well as humans, suggesting that genetic differences in regulation of the development and differentiation of adipocytes could at least partially explain the development of visceral obesity $[92,94]$.

It is noteworthy, however, that depot-specific differences have been observed even within subcutaneous adipose tissue, as demonstrated recently by Karastergiou et al, who investigated depot- and sex-dependent differences in gene expression in human abdominal and gluteal subcutaneous adipose tissue [95]. There was again strong evidence for differential regulation of mRNA expression of homeobox genes in both sexes, implying that developmentally programmed differences may contribute to the distinct phenotypic characteristics of peripheral fat [95]. Consistently, a unique expression pattern of developmental genes has been previously described by Yamamoto et al for Shox2, En1, Tbx15, Hoxa5, Hoxc8 and Hoxc9 in several subcutaneous and intra-abdominal white and brown adipose tissue depots in mice under obese and in fasting conditions (Fig. 1) [96]. With regard to gene function, it has been shown very recently that $S H O X 2$, whose expression levels in human subcutaneous adipose tissue positively correlate with visceral obesity, regulates lipolysis via increasing $A D R B 3$ expression, thus suggesting its role in adipocyte biology [97].

\section{Epigenetics and other aspects}

It should be noted that, despite recent advances in the field of high-throughput genetic analyses resulting in a number of novel polymorphisms associated with WHR, these polymorphisms can only explain a small proportion of phenotypic variance and genetic heritability in FD [30]. Therefore, other players such as non-coding RNA or DNA methylation need to be acknowledged as possible regulators of FD (Fig. 1).

Epigenetic modifications, such as DNA or histone methylation, modify long-term gene expression and seem to provide plausible mechanisms for adapting the genome to environmental circumstances. It has been shown that nutritional oscillations in certain developmental periods of life may increase susceptibility to overweight and related diseases. Perinatal programming of the genome based on prenatal and neonatal overfeeding contributes to obesity and diabetes in later life [98]. It is also increasingly appreciated that there is an association between maternal nutrition during pregnancy and intrauterine development of fetal body composition and subsequently FD later in life [99]. More importantly, body composition and adverse FD may be modifiable via nutritional intervention in the mother [99]. As mentioned above, the strong adipose tissue-specific expression patterns of genes playing a fundamental role in early development were strikingly found to be preserved from one pre-adipocyte to the next over several generations [93, 100], implying the existence of yet unknown mechanisms maintaining the expression profiles over time [101]. This is not only supported by demonstrating that white and brown adipose tissue originate from independent precursor cells [102] but also by showing distinct methylation profiles for white and brown pre-adipocytes [103]. In a genome-wide methylation analysis of eight different adipose depots in three pig breeds living within comparable environments, but displaying distinct fat levels, Li et al investigated the systematic association between anatomical locationspecific DNA methylation status of different adipose depots and obesity-related phenotypes [104]. Using methylated DNA immunoprecipitation sequencing, the authors showed that, compared with subcutaneous adipose tissue, visceral and intermuscular adipose tissue, which are the metabolic risk factors of obesity, were primarily associated with impaired inflammatory and immune responses. By presenting functionally relevant methylation differences between different adipose depots, the study supports the role of epigenetics in the regulation of FD. Epigenetic studies on animal models are now being complemented by human studies, which bring further evidence for the potential role of epigenetics in the pathophysiology of adverse FD. For instance, a recent study by Huang et al described a positive correlation between $I G F 2-H 19$ DNA methylation levels and ultrasound-derived measures of subcutaneous fat thickness in young adults [105]. Furthermore, DNA methylation levels at the LEP promoter were shown to be related to its tissue distribution [106]. As mutations in LMNA represent a major determinant of monogenic forms of altered FD, it is of interest that a recent study proposed that lamin A/C-promoter interactions specify chromatin state-dependent transcription outcomes [107]. Although these studies clearly suggest the role of epigenetic modifications in FD, the potential mechanistic chains explaining the relation between genes/SNPs, mRNA expression and gene methylation remains to be explored, and warrants further detailed analyses.

\section{Closing remarks}

Undoubtedly, and regardless of forms of altered FD, fat deposition is strongly determined by genetic factors. Whereas specific forms of disturbed FD, such as lipodystrophies, can be clearly assigned to individual genetic mutations, other 
forms, such as visceral obesity, appear to be of a polygenic nature and further influenced by environmental factors. Although genes involved in the pathophysiology of monogenic forms of altered FD may be attractive candidates in studies aimed at investigating common genetic variation and its effects on FD (as has been demonstrated for $L M N A$ variants associated with type 2 diabetes and obesity), recent GWAS on measures of FD proved to be the most efficient tool in identifying genetic loci potentially harbouring genes controlling FD (Fig. 1). The GWAS not only support the unique role of developmental genes in the regulation of FD but also point to numerous novel genes/loci whose yet unknown biological role will be challenging researchers in the near future. Even though it is unclear whether differential expression is a cause or consequence of obesity/FD pattern, it might be postulated that changes in gene/protein expression or in gene/protein function caused by genetic variants may lead to variability in FD profiles and thus link metabolic alterations to obesity (Fig. 1). It is of note that many of the WHR-associated loci have also shown associations in GWAS for metabolic traits such as fasting glucose, insulin, adiponectin levels and BMI, and with diseases such as type 2 diabetes, hypertension and coronary heart disease (ESM Table 1), so further supporting the suggestion that individuals genetically predisposed to store fat in the visceral rather than the subcutaneous depot are at higher risk of developing various metabolic complications. The challenge is now to understand the biological processes controlled by these genes leading to altered FD. For example, considering the fact that dysfunctional adipose tissue that is unable to expand through hyperplasia will lead to visceral accumulation and ectopic fat deposition, it might be hypothesised that some individuals with genetically determined dysfunctional subcutaneous adipose tissue may be more prone to storing variable amounts of fat in other ectopic depots (e.g. liver, heart, muscle, or around large vessels) depending on variation in other sets of genes (Fig. 3).

In conclusion, a better knowledge of the function of FD genes will be crucial for understanding the complex aetiology of obesity-related complications and might even pave novel paths for treatment strategies for metabolic disorders such as diabetes. A main focus of future research is therefore to unravel the yet unknown biological function of these novel 'fat distribution genes'. In addition, more accurate methods, including cardiometabolic imaging, for assessment of FD will be required to promote our knowledge in this field.

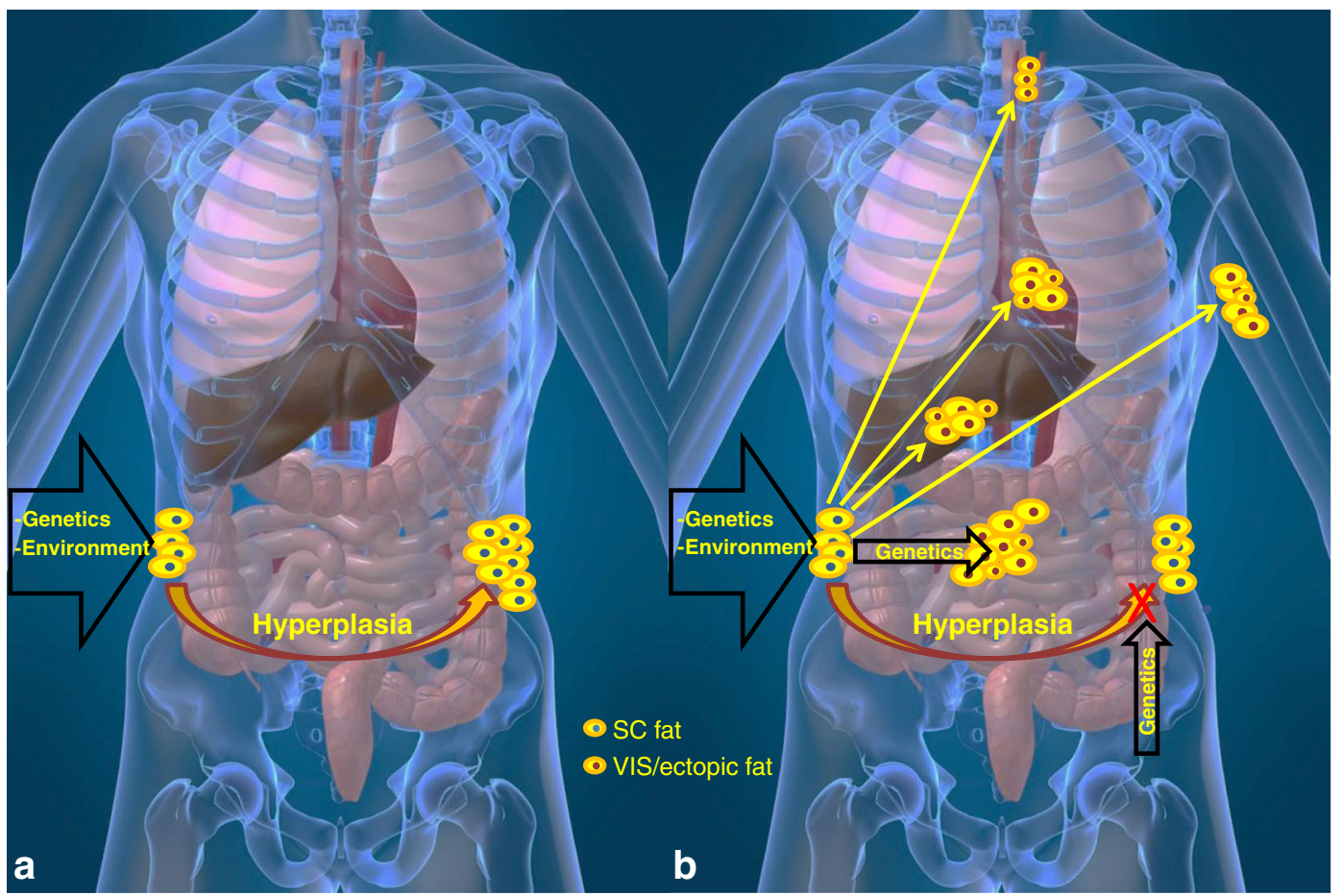

Fig. 3 (a) Functional adipose tissue expansion through hyperplasia to cover the need to store excess energy. (b) Dysfunctional adipose tissue unable to expand through hyperplasia will lead to visceral adipose tissue accumulation and to ectopic fat deposition. An excess in body fat arises in most cases from a mixture of adverse lifestyle components (e.g. low physical activity, hyper-energetic nutrition) and genetic susceptibility. In addition, expansion capacity of subcutaneous adipose tissue and storage of energy in various ectopic fat depots might be modulated by different gene sets. Thus, some individuals with genetically determined dysfunctional subcutaneous adipose tissue may be more prone to store variable amounts of fat in other ectopic depots (e.g. liver, heart, muscle or around large vessels) depending upon variation in other sets of genes. SC subcutaneous; VIS visceral 
Funding $\mathrm{PK}$ and $\mathrm{MB}$ are supported by a Collaborative Research Center granted by the DFG (Sonderforschungsbereich 1052 'Mechanismen der Adipositas'; B01, B03). YB is supported by a research grant from the DFG (BO 3147/4-1). DS is funded by the Boehringer Ingelheim Foundation. YB and PK are supported by the IFB AdiposityDiseases (K50D and K7-45 to YB; K7-56 and K7-57 to PK). IFB AdiposityDiseases is funded by the Federal Ministry of Education and Research (BMBF), Germany, FKZ: 01EO1001.

Duality of interest The authors declare that there is no duality of interest associated with this manuscript.

Contribution statement $\mathrm{DS}, \mathrm{YB}, \mathrm{MB}$ and $\mathrm{PK}$ were responsible for the conception and design of the manuscript, drafting the manuscript, revising it critically for intellectual content and approving the final version.

\section{References}

1. Van Gaal LF, Mertens IL, De Block CE (2006) Mechanisms linking obesity with cardiovascular disease. Nature 444:875-880

2. Reaven GM (2003) Importance of identifying the overweight patient who will benefit the most by losing weight. Ann Intern Med 138:420-423

3. Kloting N, Fasshauer M, Dietrich A et al (2010) Insulin-sensitive obesity. Am J Physiol Endocrinol Metab 299:E506-E515

4. Stefan N, Kantartzis K, Machann J et al (2008) Identification and characterization of metabolically benign obesity in humans. Arch Intern Med 168:1609-1616

5. Kissebah AH (1997) Central obesity: measurement and metabolic effects. Diabetes Rev 5:8-20

6. Bjorntorp P (1991) Metabolic implications of body-fat distribution. Diabetes Care 14:1132-1143

7. Matsuzawa Y, Shimomura I, Nakamura T, Keno Y, Tokunaga K (1995) Pathophysiology and pathogenesis of visceral fat obesity. Ann N Y Acad Sci 748:399-406

8. Despres JP, Nadeau A, Tremblay A et al (1989) Role of deep abdominal fat in the association between regional adipose tissue distribution and glucose tolerance in obese women. Diabetes 38: 304-309

9. Klein S, Fontana L, Young VL et al (2004) Absence of an effect of liposuction on insulin action and risk factors for coronary heart disease. N Engl J Med 350:2549-2557

10. Thorne A, Lonnqvist F, Apelman J, Hellers G, Arner P (2002) A pilot study of long-term effects of a novel obesity treatment: omentectomy in connection with adjustable gastric banding. Int J Obes Relat Metab Disord 26:193-199

11. Wajchenberg BL (2000) Subcutaneous and visceral adipose tissue: their relation to the metabolic syndrome. Endocr Rev 21:697-738

12. Bluher M (2009) Adipose tissue dysfunction in obesity. Exp Clin Endocrinol Diabetes 117:241-250

13. Speliotes EK, Massaro JM, Hoffmann U et al (2010) Fatty liver is associated with dyslipidemia and dysglycemia independent of visceral fat: the Framingham Heart Study. Hepatology 51:1979-1987

14. Foster MC, Hwang SJ, Porter SA, Massaro JM, Hoffmann U, Fox CS (2011) Fatty kidney, hypertension, and chronic kidney disease: the Framingham Heart Study. Hypertension 58:784-790

15. Fujioka S, Matsuzawa Y, Tokunaga K, Tarui S (1987) Contribution of intra-abdominal fat accumulation to the impairment of glucose and lipid metabolism in human obesity. Metabolism 36:54-59

16. Lemieux S, Prud'homme D, Bouchard C, Tremblay A, Despres JP (1996) A single threshold value of waist girth identifies normalweight and overweight subjects with excess visceral adipose tissue. Am J Clin Nutr 64:685-693
17. Lemieux I, Pascot A, Couillard C et al (2000) Hypertriglyceridemic waist: a marker of the atherogenic metabolic triad (hyperinsulinemia; hyperapolipoprotein B; small, dense LDL) in men? Circulation 102:179-184

18. Krotkiewski M, Bjorntorp P, Sjostrom L, Smith U (1983) Impact of obesity on metabolism in men and women. Importance of regional adipose tissue distribution. J Clin Invest 72:1150-1162

19. Goss AM, Darnell BE, Brown MA, Oster RA, Gower BA (2012) Longitudinal associations of the endocrine environment on fat partitioning in postmenopausal women. Obesity (Silver Spring) 20:939-944

20. Seidell JC, Bjorntorp P, Sjostrom L, Kvist H, Sannerstedt R (1990) Visceral fat accumulation in men is positively associated with insulin, glucose, and C-peptide levels, but negatively with testosterone levels. Metabolism 39:897-901

21. Bouchard C, Tremblay A, Despres JP et al (1990) The response to long-term overfeeding in identical twins. N Engl J Med 322: 1477-1482

22. Smith SR, Zachwieja JJ (1999) Visceral adipose tissue: a critical review of intervention strategies. Int J Obes Relat Metab Disord 23: 329-335

23. Ronn M, Kullberg J, Karlsson H et al (2013) Bisphenol A exposure increases liver fat in juvenile fructose-fed Fischer 344 rats. Toxicology 303:125-132

24. Malik VS, Popkin BM, Bray GA, Despres JP, Hu FB (2010) Sugarsweetened beverages, obesity, type 2 diabetes mellitus, and cardiovascular disease risk. Circulation 121:1356-1364

25. Bjorntorp P (2001) Do stress reactions cause abdominal obesity and comorbidities? Obes Rev 2:73-86

26. Souren NY, Paulussen AD, Loos RJ et al (2007) Anthropometry, carbohydrate and lipid metabolism in the East Flanders Prospective Twin Survey: heritabilities. Diabetologia 50:2107-2116

27. Mills GW, Avery PJ, McCarthy MI et al (2004) Heritability estimates for beta cell function and features of the insulin resistance syndrome in UK families with an increased susceptibility to type 2 diabetes. Diabetologia 47:732-738

28. Selby JV, Newman B, Quesenberry CP Jr et al (1990) Genetic and behavioral influences on body fat distribution. Int J Obes 14:593-602

29. Rose KM, Newman B, Mayer-Davis EJ, Selby JV (1998) Genetic and behavioral determinants of waist-hip ratio and waist circumference in women twins. Obes Res 6:383-392

30. Heid IM, Jackson AU, Randall JC et al (2010) Meta-analysis identifies 13 new loci associated with waist-hip ratio and reveals sexual dimorphism in the genetic basis of fat distribution. Nat Genet 42:949-960

31. Perusse L, Despres JP, Lemieux S, Rice T, Rao DC, Bouchard C (1996) Familial aggregation of abdominal visceral fat level: results from the Quebec Family Study. Metabolism 45:378-382

32. Malis C, Rasmussen EL, Poulsen P et al (2005) Total and regional fat distribution is strongly influenced by genetic factors in young and elderly twins. Obes Res 13:2139-2145

33. Peeters MW, Beunen GP, Maes HH et al (2007) Genetic and environmental determination of tracking in subcutaneous fat distribution during adolescence. Am J Clin Nutr 86:652-660

34. Ersek RA, Bell HN, Salisbury AV (1994) Serial and superficial suction for steatopygia (Hottentot bustle). Aesthet Plast Surg 18: 279-282

35. Garg A (2011) Clinical review\#: Lipodystrophies: genetic and acquired body fat disorders. J Clin Endocrinol Metab 96: 3313-3325

36. Yang W, Thein S, Guo X et al (2013) Seipin differentially regulates lipogenesis and adipogenesis through a conserved core sequence and an evolutionarily acquired C-terminus. Biochem J 452:37-44 
37. Agarwal AK, Arioglu E, De Almeida S et al (2002) AGPAT2 is mutated in congenital generalized lipodystrophy linked to chromosome 9q34. Nat Genet 31:21-23

38. Razani B, Combs TP, Wang XB et al (2002) Caveolin-1-deficient mice are lean, resistant to diet-induced obesity, and show hypertriglyceridemia with adipocyte abnormalities. J Biol Chem 277: 8635-8647

39. Aboulaich N, Chui PC, Asara JM, Flier JS, Maratos-Flier E (2011) Polymerase I and transcript release factor regulates lipolysis via a phosphorylation-dependent mechanism. Diabetes 60:757-765

40. Garg A, Peshock RM, Fleckenstein JL (1999) Adipose tissue distribution pattern in patients with familial partial lipodystrophy (Dunnigan variety). J Clin Endocrinol Metab 84:170-174

41. Shackleton S, Lloyd DJ, Jackson SNJ et al (2000) LMNA, encoding lamin $\mathrm{A} / \mathrm{C}$, is mutated in partial lipodystrophy. Nat Genet 24 : $153-156$

42. Cao H, Hegele RA (2000) Nuclear lamin A/C R482Q mutation in Canadian kindreds with Dunnigan-type familial partial lipodystrophy. Hum Mol Genet 9:109-112

43. Wojtanik KM, Edgemon K, Viswanadha S et al (2009) The role of LMNA in adipose: a novel mouse model of lipodystrophy based on the Dunnigan-type familial partial lipodystrophy mutation. J Lipid Res 50:1068-1079

44. Wegner L, Andersen G, Sparsø T et al (2007) Common variation in LMNA increases susceptibility to type 2 diabetes and associates with elevated fasting glycemia and estimates of body fat and height in the general population: studies of 7,495 Danish Whites. Diabetes 56:694-698

45. Hegele RA, Cao H, Harris SB, Zinman B, Hanley AJ, Anderson CM (2000) Genetic variation in LMNA modulates plasma leptin and indices of obesity in aboriginal Canadians. Physiol Genomics 3:39-44

46. Steinle NI, Kazlauskaite R, Imumorin IG et al (2004) Variation in the lamin $\mathrm{A} / \mathrm{C}$ gene: associations with metabolic syndrome. Arterioscler Thromb Vasc Biol 24:1708-1713

47. Toy BR (2003) Familial multiple lipomatosis. Dermatol Online J 9:9

48. Schoenmakers EF, Wanschura S, Mols R, Bullerdiek J, Van den BH, Van de Ven WJ (1995) Recurrent rearrangements in the high mobility group protein gene, HMGI-C, in benign mesenchymal tumours. Nat Genet 10:436-444

49. Markowski DN, Thies HW, Gottlieb A, Wenk H, Wischnewsky M, Bullerdiek J (2013) HMGA2 expression in white adipose tissue linking cellular senescence with diabetes. Genes Nutr 8:449-456

50. Fedele M, Battista S, Manfioletti G, Croce CM, Giancotti V, Fusco A (2001) Role of the high mobility group A proteins in human lipomas. Carcinogenesis 22:1583-1591

51. Battista S, Fidanza V, Fedele M et al (1999) The expression of a truncated HMGI-C gene induces gigantism associated with lipomatosis. Cancer Res 59:4793-4797

52. Zhou X, Benson KF, Ashar HR, Chada K (1995) Mutation responsible for the mouse pygmy phenotype in the developmentally regulated factor HMGI-C. Nature 376:771-774

53. Pasquali D, Pierantoni GM, Fusco A et al (2004) Fenofibrate increases the expression of high mobility group AT-hook 2 (HMGA2) gene and induces adipocyte differentiation of orbital fibroblasts from Graves' ophthalmopathy. J Mol Endocrinol 33: 133-143

54. Widen E, Lehto M, Kanninen $\mathrm{T}$, Walston J, Shuldiner AR, Groop LC (1995) Association of a polymorphism in the beta 3 -adrenergic-receptor gene with features of the insulin resistance syndrome in Finns. N Engl J Med 333:348-351

55. Pouliot MC, Despres JP, Dionne FT et al (1994) ApoB-100 gene EcoRI polymorphism. Relations to plasma lipoprotein changes associated with abdominal visceral obesity. Arterioscler Thromb $14: 527-533$
56. Rebuffe-Scrive M, Lundholm K, Bjorntorp P (1985) Glucocorticoid hormone binding to human adipose tissue. Eur J Clin Invest 15: 267-271

57. Fried SK, Russell CD, Grauso NL, Brolin RE (1993) Lipoprotein lipase regulation by insulin and glucocorticoid in subcutaneous and omental adipose tissues of obese women and men. J Clin Invest 92: 2191-2198

58. Alessi MC, Peiretti F, Morange P, Henry M, Nalbone G, Juhan-Vague I (1997) Production of plasminogen activator inhibitor 1 by human adipose tissue: possible link between visceral fat accumulation and vascular disease. Diabetes 46: $860-867$

59. Kloting N, Graham TE, Berndt J et al (2007) Serum retinol-binding protein is more highly expressed in visceral than in subcutaneous adipose tissue and is a marker of intra-abdominal fat mass. Cell Metab 6:79-87

60. Montague CT, Prins JB, Sanders L et al (1998) Depot-related gene expression in human subcutaneous and omental adipocytes. Diabetes 47:1384-1391

61. Fried SK, Bunkin DA, Greenberg AS (1998) Omental and subcutaneous adipose tissues of obese subjects release interleukin-6: depot difference and regulation by glucocorticoid. J Clin Endocrinol Metab 83:847-850

62. Lefebvre AM, Laville M, Vega N et al (1998) Depot-specific differences in adipose tissue gene expression in lean and obese subjects. Diabetes 47:98-103

63. Parikh H, Groop L (2004) Candidate genes for type 2 diabetes. Rev Endocr Metab Disord 5:151-176

64. Bottcher Y, Unbehauen H, Kloting N et al (2009) Adipose tissue expression and genetic variants of the bone morphogenetic protein receptor 1A gene (BMPR1A) are associated with human obesity. Diabetes 58:2119-2128

65. Kovacs P, Geyer M, Berndt J et al (2007) Effects of genetic variation in the human retinol binding protein-4 gene (RBP4) on insulin resistance and fat depot-specific mRNA expression. Diabetes 56: 3095-3100

66. Schleinitz D, Kloting N, Korner A et al (2010) Effect of genetic variation in the human fatty acid synthase gene (FASN) on obesity and fat depot-specific mRNA expression. Obesity 18:1218-1225

67. Schleinitz D, Kloting N, Bottcher Y et al (2011) Genetic and evolutionary analyses of the human bone morphogenetic protein receptor 2 (BMPR2) in the pathophysiology of obesity. PLoS One 6:e16155

68. Yang X, Smith U (2007) Adipose tissue distribution and risk of metabolic disease: does thiazolidinedione-induced adipose tissue redistribution provide a clue to the answer? Diabetologia 50: $1127-1139$

69. Kodama N, Tahara N, Tahara A et al (2013) Effects of pioglitazone on visceral fat metabolic activity in impaired glucose tolerance or type 2 diabetes mellitus. J Clin Endocrinol Metab 98(11):4438-4445

70. Ristow M, Muller-Wieland D, Pfeiffer A, Krone W, Kahn CR (1998) Obesity associated with a mutation in a genetic regulator of adipocyte differentiation. N Engl J Med 339:953-959

71. Passaro A, Dalla NE, Marcello C et al (2011) PPARgamma Pro12Ala and ACE ID polymorphisms are associated with BMI and fat distribution, but not metabolic syndrome. Cardiovasc Diabetol 10:112

72. Kim KS, Choi SM, Shin SU, Yang HS, Yoon Y (2004) Effects of peroxisome proliferator-activated receptor-gamma 2 Pro12Ala polymorphism on body fat distribution in female Korean subjects. Metabolism 53:1538-1543

73. Peeters AV, Beckers S, Verrijken A et al (2008) Association of SIRT1 gene variation with visceral obesity. Hum Genet 124:431-436 
74. Chambers JC, Elliott P, Zabaneh D et al (2008) Common genetic variation near MC4R is associated with waist circumference and insulin resistance. Nat Genet 40:716-718

75. Heard-Costa NL, Zillikens MC, Monda KL et al (2009) NRXN3 is a novel locus for waist circumference: a genome-wide association study from the CHARGE Consortium. PLoS Genet 5:e1000539

76. Speliotes EK, Willer CJ, Berndt SI et al (2010) Association analyses of 249,796 individuals reveal 18 new loci associated with body mass index. Nat Genet 42:937-U53

77. Lindgren CM, Heid IM, Randall JC et al (2009) Genome-wide association scan meta-analysis identifies three loci influencing adiposity and fat distribution. PLoS Genet 5:e1000508

78. Berndt SI, Gustafsson S, Magi R et al (2013) Genome-wide metaanalysis identifies 11 new loci for anthropometric traits and provides insights into genetic architecture. Nat Genet 45:501-512

79. Norris JM, Langefeld CD, Talbert ME et al (2009) Genome-wide association study and follow-up analysis of adiposity traits in Hispanic Americans: the IRAS Family Study. Obesity (Silver Spring) 17:19321941

80. Fox CS, Liu Y, White CC et al (2012) Genome-wide association for abdominal subcutaneous and visceral adipose reveals a novel locus for visceral fat in women. PLoS Genet 8:e1002695

81. Liu CT, Monda KL, Taylor KC et al (2013) Genome-wide association of body fat distribution in African ancestry populations suggests new loci. PLoS Genet 9:e1003681

82. Hotta K, Kitamoto A, Kitamoto T et al (2013) Replication study of 15 recently published loci for body fat distribution in the Japanese population. J Atheroscler Thromb 20:336-350

83. Fox CS, White CC, Lohman K et al (2012) Genome-wide association of pericardial fat identifies a unique locus for ectopic fat. PLoS Genet 8:e1002705

84. Randall JC, Winkler TW, Kutalik Z et al (2013) Sex-stratified genome-wide association studies including 270,000 individuals show sexual dimorphism in genetic loci for anthropometric traits. PLoS Genet 9:e1003500

85. Zillikens MC, Yazdanpanah M, Pardo LM et al (2008) Sex-specific genetic effects influence variation in body composition. Diabetologia 51:2233-2241

86. Shmueli O, Horn-Saban S, Chalifa-Caspi V et al (2003) GeneNote: whole genome expression profiles in normal human tissues. C R Biol 326:1067-1072

87. Sandholt CH, Hansen T, Pedersen O (2012) Beyond the fourth wave of genome-wide obesity association studies. Nutr Diabetes 2:e37

88. Schleinitz D, Kloting N, Lindgren CM et al (2013) Fat depotspecific mRNA expression of novel loci associated with waist-hip ratio. Int J Obes 38(1):120-125

89. Cooney GJ, Lyons RJ, Crew AJ et al (2004) Improved glucose homeostasis and enhanced insulin signalling in Grb14-deficient mice. EMBO J 23:582-593

90. Cariou B, Capitaine N, Le MV et al (2004) Increased adipose tissue expression of Grb14 in several models of insulin resistance. FASEB J 18:965-967
91. Holt LJ, Lyons RJ, Ryan AS et al (2009) Dual ablation of Grb10 and Grb14 in mice reveals their combined role in regulation of insulin signaling and glucose homeostasis. Mol Endocrinol 23:1406-1414

92. Gesta S, Bluher M, Yamamoto Y et al (2006) Evidence for a role of developmental genes in the origin of obesity and body fat distribution. Proc Natl Acad Sci U S A 103:6676-6681

93. Tchkonia T, Lenburg M, Thomou $\mathrm{T}$ et al (2007) Identification of depot-specific human fat cell progenitors through distinct expression profiles and developmental gene patterns. Am J Physiol Endocrinol Metab 292:E298-E307

94. Gesta S, Tseng YH, Kahn CR (2007) Developmental origin of fat: tracking obesity to its source. Cell 131:242-256

95. Karastergiou K, Fried SK, Xie H et al (2013) Distinct developmental signatures of human abdominal and gluteal subcutaneous adipose tissue depots. J Clin Endocrinol Metab 98:362-371

96. Yamamoto Y, Gesta S, Lee KY, Tran TT, Saadatirad P, Kahn CR (2010) Adipose depots possess unique developmental gene signatures. Obesity (Silver Spring) 18:872-878

97. Lee KY, Yamamoto Y, Boucher J et al (2013) Shox2 is a molecular determinant of depot-specific adipocyte function. Proc Natl Acad Sci U S A 110:11409-11414

98. Plagemann A (2005) Perinatal programming and functional teratogenesis: impact on body weight regulation and obesity. Physiol Behav 86:661-668

99. Blumfield ML, Hure AJ, MacDonald-Wicks LK et al (2012) Dietary balance during pregnancy is associated with fetal adiposity and fat distribution. Am J Clin Nutr 96:1032-1041

100. Caserta F, Tchkonia T, Civelek VN et al (2001) Fat depot origin affects fatty acid handling in cultured rat and human preadipocytes. Am J Physiol Endocrinol Metab 280:E238-E247

101. Pinnick KE, Karpe F (2011) DNA methylation of genes in adipose tissue. Proc Nutr Soc 70:57-63

102. Seale P, Bjork B, Yang W et al (2008) PRDM16 controls a brown fat/skeletal muscle switch. Nature 454:961-967

103. Sakamoto H, Suzuki M, Abe T et al (2007) Cell type-specific methylation profiles occurring disproportionately in CpG-less regions that delineate developmental similarity. Genes Cells 12:1123-1132

104. Li M, Wu H, Wang T et al (2012) Co-methylated genes in different adipose depots of pig are associated with metabolic, inflammatory and immune processes. Int J Biol Sci 8:831-837

105. Huang RC, Galati JC, Burrows S et al (2012) DNA methylation of the IGF2/H19 imprinting control region and adiposity distribution in young adults. Clin Epigenetics 4:21

106. Marchi M, Lisi S, Curcio M et al (2011) Human leptin tissue distribution, but not weight loss-dependent change in expression, is associated with methylation of its promoter. Epigenetics 6: $1198-1206$

107. Lund E, Oldenburg AR, Delbarre E et al (2013) Lamin A/Cpromoter interactions specify chromatin state-dependent transcription outcomes. Genome Res 23:1580-1589 